\title{
ON THE REDUCIBILITY OF LINEAR GROUPS*
}

BY

\section{LEONARD EUGENE DICKSON}

The object of this note is a two-fold generalization of Loewr's theorem proved in these Transactions, vol. 4, pp. 171-177. His theorem may be conveniently stated as follows: If $R$ is the domain of all real numbers and $C$ the domain of all complex numbers, any group of linear homogeneous transformations with coefficients in $R$ which is irreducible in $R$, but reducible in $C$, can be transformed linearly into a decomposable group $\left(\begin{array}{cc}G & 0 \\ 0 & . \bar{G}\end{array}\right)$, where $G$ and $\bar{G}$ are two groups irreducible in $C$, with coefficients not all in $R$, such that the coefficients in every transformation of $\bar{G}$ are the conjugate imaginaries of the corresponding coefficients for $G$.

In seeking a generalization, we note that the domain $C$ may be considered as derived from $R$ by the adjunction of a root $i$ of the quadratic equation $x^{2}+1=0$ belonging to and irreducible in $R$. For the generalization, $R$ is replaced by a general domain $F$ (or field not having a modulus) and $R(i)$ is replaced by the domain $F\left(\rho_{0}\right)$ given by the extension of $F$ by the adjunction of a root $\rho_{0}$ of an equation $f(x)=0$ of degree $r$ belonging to and irreducible in $F$. The generalization will therefore be two-fold. Let the roots of $f(x)=0$ be $\rho_{0}, \rho_{1}, \cdots, \rho_{r-1}$. If $G_{11}$ is a group of transformations with coefficients $C_{i j}\left(\rho_{0}\right)$ in the domain $F\left(\rho_{0}\right)$, let $G_{11}^{(s)}$ denote the group of transformations with the coefficients $C_{i j}\left(\rho_{s}\right)$; in particular, $G_{11}^{(0)}=G_{11}$. The coefficients of $G_{11}, G_{11}^{\prime}, \ldots, G_{11}^{(r-1)}$ are thus conjugate with respect to $F$. The generalized theorem is as follows :

Let $G$ be a group of linear homogeneous transformations with coefficients in a domain $F$, such that $G$ is irreducible in $F$ but is reducible in the domain $F\left(\rho_{0}\right)$ given by the extension of $F$ by the adjunction of a root $\rho_{0}$ of an equation belonging to and irreducible in $F$ and having as its roots $\rho_{0}, \rho_{1}, \ldots, \rho_{r-1}$. Then $G$ can be transformed linearly into a decomposable group *

\footnotetext{
* Presented to the Society at the Boston summer meeting, August 31-September 1, 1903. Received for publication April 27, 1903.

$\dagger$ When the irreducible equation is a normal equation, the groups $G_{11}^{(s)}(s=0,1, \cdots, r-1)$ are all irreducible in the same (normal) domain. LokWY's case furnishes an example.
} 


$$
\begin{aligned}
& \begin{array}{lllll}
G_{11} & 0 & 0 & \ldots & 0
\end{array} \\
& \begin{array}{lllll}
0 & G_{11}^{\prime} & 0 & \ldots & 0
\end{array} \\
& \begin{array}{lllll}
0 & 0 & G_{11}^{\prime \prime} & \cdots & 0
\end{array} \\
& \begin{array}{lllll}
0 & 0 & 0 & \ldots & G_{11}^{(r-1)}
\end{array}
\end{aligned}
$$

where $G_{11}^{(s)}$ is a group irreducible in $F\left(\rho_{0}\right)$ with coefficients not all in $F$, and $G_{11}, G_{11}^{\prime}, \ldots, G_{11}^{(r-1)}$ are conjugate with respect to $F$.

The proof starts as in LoEwY, $§ 1$. The first variation* occurs at the bottom of p. 173 ; we now take $r$-fold decomposable matrices

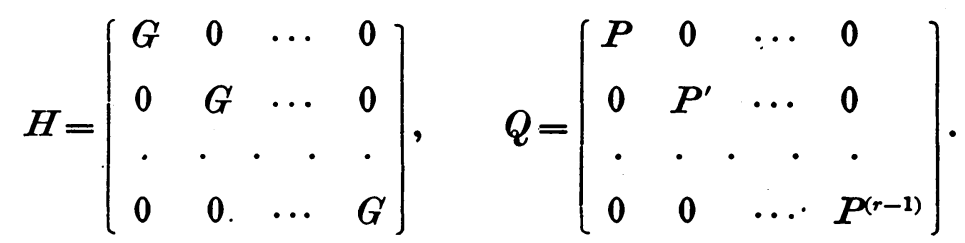

Corresponding changes are to be made in the first two statements on p. 174 . Thus, the diagonal groups in (6) are to be replaced by

$$
G_{11}, G_{22}, G_{11}^{\prime}, G_{22}^{\prime}, G_{11}^{\prime \prime}, G_{22}^{\prime \prime}, \cdots, G_{11}^{(r-1)}, G_{22}^{(r-1)} \text {. }
$$

In place of the transformation $\dagger(7)$, we have

$$
y_{j k}=\sum_{i=1}^{n} C_{k i}^{(j)} y_{j i}^{*} \quad(k=1, \cdots, n ; j=0, \cdots, r-1),
$$

where $C_{k i}^{(j)}$ is a rational function of $\rho_{j}$ with coefficients in $F$, and

$$
C_{k i}^{(j)}=0 \quad(k=1, \cdots, m ; i=m+1, \cdots, n ; j=0, \cdots, r-1) .
$$

Introduce two pairs each of $r n$ new variables defined by

$$
\begin{array}{ll}
y_{s k}=Y_{0 k}+\rho_{s} Y_{1 k}+\rho_{s}^{2} Y_{2 k}+\cdots+\rho_{s}^{r-1} Y_{r-1 k} & \left(\begin{array}{l}
s=0, \cdots, r-1 \\
k=1, \cdots, n
\end{array}\right) . \\
y_{s k}^{*}=Y_{0 k}^{*}+\rho_{s} Y_{1 k}^{*}+\rho_{s}^{2} Y_{2 k}^{*}+\cdots+\rho_{s}^{r-1} Y_{r-1 k}^{*} &
\end{array}
$$

This may be done since the determinant

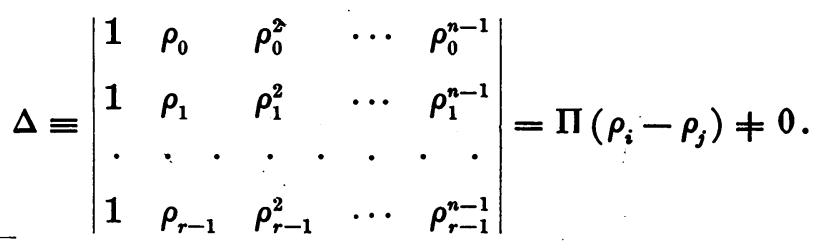

\footnotetext{
* The statement on p. 173, lines 7-8, is apparently not used later ; a proof follows readily from the main theorem under consideration.

† LokwY's notation is unwieldy even in his simple case. I write $y_{0 k}, y_{1 k}$ for his $y_{k}, z_{k}$. The transformed variables are marked * instead of being primed.
}

Trans. Am. Math. Soc. 29 
Solving $\left(8^{\prime}\right)$ for fixed $k$, while $s=0, \cdots, r-1$, we get

where

$$
\Delta Y_{t k}=\sum_{s=0}^{r-1}(-1)^{t} D_{t s} y_{s k} \quad(t=0,1, \cdots, r-1),
$$

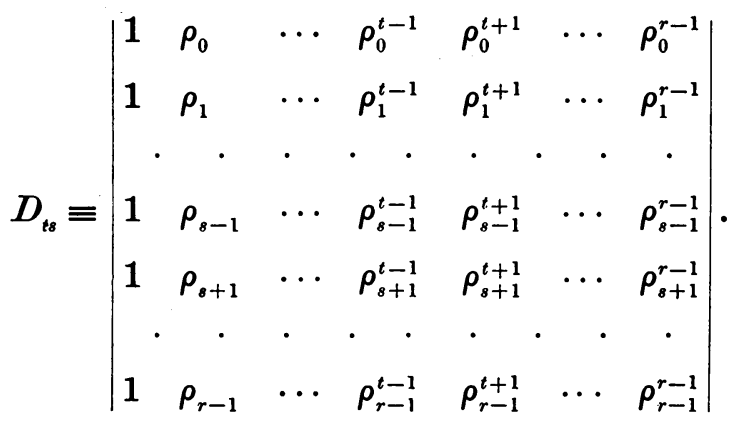

Substituting for $y_{s k}$ in $(e)$ its value from $\left(7^{\prime}\right)$ and then eliminating $y_{s i}^{*}$ by $\left(8_{1}^{\prime}\right)$, we obtain

where

$$
Y_{t k}=\sum_{\substack{i=1, \ldots, n \\ l=0, \ldots, r-1}} \alpha_{l i}^{t k} Y_{l i}^{*} \quad(k=1, \cdots n ; t=0, \cdots r-1),
$$

$$
\alpha_{l i}^{t k} \equiv \frac{(-1)^{t}}{\Delta} \sum_{s=0}^{r-1}(-1)^{s} D_{t s} C_{k i}^{(s)} \rho_{s}^{l}
$$

The coefficients of transformation (9) belong to the domain $F$. It suffices to show that each $\alpha_{l i}^{t k}$ is unaltered by the interchange of $\rho_{0}$ with $\rho_{j}$ ( $j$ being any one of the series $1,2, \ldots, r-1)$, since it is then a symmetric function of $\rho_{0}, \rho_{1}, \cdots, \rho_{r-1}$ with coefficients in $F$. To show that, for example, it is unaltered by the interchange of $\rho_{0}$ with $\rho_{1}$, we note that under this interchange, $D_{t 0}$ and $D_{t 1}$ are interchanged, $D_{t s}(s>1)$ is changed into $-D_{t s}$ while $C_{k i}^{(0)} \equiv C_{k i}\left(\rho_{0}\right)$ and $C_{k i}^{(1)} \equiv C_{k i}\left(\rho_{1}\right)$ are interchanged, and $C_{k i}^{(s)}(s>1)$ is unaltered. Hence the factor of $\alpha$ given by the sum is changed in sign; likewise the factor $1 / \Delta$.

Moreover, from $\left(7_{a}\right)$ follows at once

$$
\alpha_{l i}^{t k}=0 \quad(i=m+1, \therefore, n ; k=1, \cdots, m ; t, l=0,1, \cdots, r-1) .
$$

The group of transformations (9) is therefore of LoEwY's form (10), $\bar{H}_{11}$ being always a matrix of $r m$ rows and $r m$ columns. The proof is then readily completed as in LoEwY's case (bottom of p. 175 and 176).

The University of Chicago, April 27, 1903. 